\title{
ANÁLISE DOS PRINCIPAIS FATORES DE RISCO PREEXISTENTES EM PACIENTES DIAGNOSTICADOS COM A COVID-19 NO AMAPÁ, AMAZÔNIA, BRASIL
}

\section{ARTIGO ORIGINAL}

OLIVEIRA, Arthur Aires de ${ }^{1}$, MOREIRA, Danilo José Silva², PIMENTEL, Jhon Allyson Sena $^{3}$, COSTA, Pedro Henrique de Magalhães ${ }^{4}$, DIAS, Claudio Alberto Gellis de Mattos $^{5}$, ARAÚJO, Maria Helena Mendonça de ${ }^{6}$, OLIVEIRA, Euzébio de ${ }^{7}$, DENDASCK, Carla Viana ${ }^{8}$, SOUZA, Keulle Oliveira da ${ }^{9}$, FECURY, Amanda Alves $^{10}$

OLIVEIRA, Arthur Aires de. Et al. Análise dos principais fatores de risco preexistentes em pacientes diagnosticados com a COVID-19 no Amapá, Amazônia, Brasil. Revista Científica Multidisciplinar Núcleo do Conhecimento. Ano 06, Ed. 06, Vol. 17, pp. 56-72. Junho de 2021. ISSN: 2448-0959, Link de acesso: https://www.nucleodoconhecimento.com.br/saude/risco-preexistentes, DOI: 10.32749/nucleodoconhecimento.com.br/saude/risco-preexistentes

\section{RESUMO}

\footnotetext{
${ }^{1}$ Acadêmico de Medicina. Universidade Federal do Amapá (UNIFAP).

${ }^{2}$ Acadêmico de Medicina. Universidade Federal do Amapá (UNIFAP).

${ }^{3}$ Acadêmico de Medicina. Universidade Federal do Amapá (UNIFAP).

${ }^{4}$ Acadêmico de Medicina. Universidade Federal do Amapá (UNIFAP).

${ }^{5}$ Biólogo, Doutor em Teoria e Pesquisa do Comportamento, Professor e pesquisador do Curso de Licenciatura em Química do Instituto de Ensino Básico, Técnico e Tecnológico do Amapá (IFAP) e do Programa de Pósgraduação em Educação Profissional e Tecnológica (PROFEPT IFAP).

${ }^{6}$ Médica, Mestra em Ensino e Ciências da Saúde, Professora e pesquisadora do Curso de Medicina do Campus Macapá, Universidade Federal do Amapá (UNIFAP).

${ }^{7}$ Biólogo, Doutor em Doenças Tropicais, Professor e pesquisador do Curso de Educação Física, Universidade Federal do Pará (UFPA).

${ }^{8}$ Teóloga, Doutora em Psicanálise Clínica. Atua há 15 anos com Metodologia Científica ( Método de Pesquisa) na Orientação de Produção Científica de Mestrandos e Doutorandos. Especialista em Pesquisas de Mercado e Pesquisas voltadas a área da Saúde. Doutoranda em Comunicação e Semiótica (PUC SP).

${ }^{9}$ Cientista Social, Especialista em Gestão e Docência do Ensino Superior, Mestranda em Estudos Antrópicos na Amazônia (UFPA).

${ }^{10}$ Biomédica, Doutora em Doenças Tropicais, Professora e pesquisadora do Curso de Medicina do Campus Macapá, Universidade Federal do Amapá (UNIFAP), Pró-reitora de Pesquisa e Pós-Graduação (PROPESPG) da Universidade Federal do Amapá (UNIFAP).
} 
A COVID-19 (Coronavirus Disease 2019) é uma infecção causada pelo vírus SARSCoV-2, com primeiros casos notificados em dezembro de 2019 na cidade de Wuhan. Constatou-se que em pacientes com comorbidades, há maior risco de complicações e mortalidade em caso de infecção. A doença foi considerada pela Organização Mundial de Saúde em 2020 com uma emergência de saúde pública e, no Brasil, o Ministério da Saúde implementou medidas para monitoramento epidemiológico dos casos registrados nas diferentes unidades federativas do país. Dentre estas, o Amapá assume níveis elevados de incidência e mortalidade. Este estudo visa analisar os principais fatores de risco prévios constatados em pacientes com COVID-19 no Estado do Amapá. Para isso, utilizou-se dados secundários disponíveis no Painel Coronavirus Amapá, através de uma busca realizada no dia 22 de maio de 2020. $\mathrm{Na}$ plataforma, colheu-se dados sobre o número de pacientes diagnosticados com a COVID-19, assim como o número de óbitos em virtude da doença, com avaliação conjunta em cada tópico das comorbidades constatadas. Após agrupar esses dados em planilhas do Microsoft Excel, uma análise quantitativa e descritiva destes foi feita. No Estado, registrou-se um total de 28.927 pacientes diagnosticados com COVID-19, notificados até o dia da coleta. Cerca de 1,31\% (378 casos) destes possuíam um fator de risco que poderiam suscetibilizá-los a desenvolver complicações decorrentes da COVID-19, sendo diabetes e doenças cardíacas crônicas as condições com maior número de registros. Ainda no Estado do Amapá, 420 óbitos em pacientes com COVID-19 foram constatados. Desse total, $75,71 \%$ (318 casos) possuíam fatores de risco prévios para maior risco de complicações devido à COVID-19, com hipertensão e diabetes sendo as condições predominantes. Com o estudo, percebeu-se no Amapá, que grande parte da população de pacientes diagnosticados com a doença e que faleceram apresentavam um histórico de fator de risco para a COVID-19. Em virtude da notificação de fatores de risco no quadro de óbito que não foram registrados no quadro de pacientes diagnosticados, assim como da inespecificidade inerente ao quadro dos pacientes monitorados pelos mecanismos sanitários do Estado, não foi possível realizar uma análise mais detalhada acerca do grau de complicação e taxa de letalidade gerados por determinado fator de risco e sua associação à infecção por coronavírus. 
Palavras-chave: COVID-19, Fatores de risco, Comorbidade.

\section{INTRODUÇÃO}

Na primeira metade do mês de dezembro de 2019, o primeiro caso de pneumonia de origem desconhecida fora relatado em Wuhan, província de Hubei, China. Posteriormente, um surto de casos de pneumonia por Síndrome Respiratória Aguda Grave (SRAG) assolou a província, chamando atenção da Organização Mundial da Saúde (OMS), a qual, partindo da circunscrição das suas atribuições, declarou que a situação se tratava de uma epidemia causada por um subtipo novo de vírus pertencente à família Coronaviridae, o coronavírus-2, constituindo Emergência de Saúde Pública de Importância Internacional (ESPII), em 30 de janeiro de 2020 (COSTA et al., 2020a; FERRARI, 2020; GAUTRET et al., 2020; GUAN et al., 2020; LI et al., 2020; LIMA et al., 2020; MARTINS-CHAVES; GOMES; GOMEZ, 2020; OLIVEIRA et al., 2020; RENU; PRASANNA; GOPALAKRISHNAN, 2020; RODRíGUEZ-COLA et al., 2020; TAY et al., 2020; ZHAl et al., 2020).

Em fevereiro de 2020, o Diretor Geral da OMS, nomeou a enfermidade como doença do coronavírus-19 (COVID-19), sendo uma infecção causada pelo SARS-CoV-2 (da expressão em inglês: Severe Acute Respiratory Syndrome Coronavirus 2), um dos sete subtipos de coronavírus já conhecidos (COSTA et al., 2020a; RENU; PRASANNA; GOPALAKRISHNAN, 2020; HONG et al., 2020).

Sob a perspectiva brasileira, a infecção por COVID-19 foi declarada pelo Ministério da Saúde (MS) como Emergência de Saúde Pública de Importância Nacional (ESPIN) no dia 03 de fevereiro de 2020, e teve seu primeiro caso confirmado no Brasil em 26 de fevereiro (OLIVEIRA et al., 2020; OLIVEIRA; LUCAS; IQUIAPAZA, 2020). Como medida de apoio ao Sistema de Saúde, o Departamento de Informática do Sistema Único de Saúde (DATASUS), mediante o Plano de Contingência, agiu provendo assistência virtual à população através do aplicativo "Coronavírus SUS" (BRASIL, 2020a). 
No Brasil, o avanço da pandemia é monitorado majoritariamente pelo MS através de índices estatísticos que refletem o status de cada unidade federativa (UF), sendo que o Estado do Amapá assume índices epidemiológicos elevados, em especial quanto ao coeficiente de incidência de COVID-19 (BRASIL, 2020c).

Até o dia 17 de julho de 2020 o Amapá liderava o Norte brasileiro no ranking do Coeficiente de Incidência de COVID-19 por UF de notificação (número de casos confirmados por 100 mil habitantes), cujo valor era de 3.637,4 sendo seguido por Roraima, com 3.327,1, que por sua vez era subsequenciada pelo Estado do Amazonas, com 1962,0. Quanto ao Coeficiente de Mortalidade de COVID-19 (número de mortes por 100 mil habitantes), o Amapá posicionava-se em $7^{\circ}$ lugar $(55,2)$ (BRASIL, 2020c).

Fatores de risco dizem respeito a quaisquer condições que prejudiquem a resposta imunitária do organismo frente a uma enfermidade. Dessa forma, tais fatores incluem tanto as comorbidades, isto é, doenças crônicas previamente instaladas e que atuam concomitantemente no organismo do paciente, como também particularidades dos indivíduos, quais sejam: condição puerperal, tabagismo, etilismo, dentre outras (PIRES; CARVALHO; XAVIER, 2020; ENGIN; ENGIN; ENGIN, 2020; KHALIQ; PHOSWA, 2020).

Estudos sugerem que pacientes infectados com COVID-19 que possuam comorbidades apresentam maior vulnerabilidade imunológica bem como maior taxa de letalidade quando comparados a pacientes afetados somente pelo vírus. Entre as principais comorbidades listadas, incluem-se hipertensão, diabetes, cardiopatias e doenças crônicas do sistema respiratório (COSTA et al., 2020a; FERRARI, 2020; MARTINS-CHAVES; GOMES; GOMEZ, 2020; RENU; PRASANNA; GOPALAKRISHNAN, 2020; YANG et al., 2020).

Frente ao panorama mundial e diante do status do Estado do Amapá, torna-se imprescindível realizar análises, estudos e fomentos em pesquisas de cunho científico, a fim que se estabeleçam manejos adequados aos pacientes acometidos por doenças subjacentes e/ou condições que representem potenciais fatores de risco 
ao prognóstico de pacientes diagnosticados com SARS-CoV-2, visando atenuar as complicações apresentadas por eles (RENU; PRASANNA; GOPALAKRISHNAN, 2020; YANG et al., 2020).

\section{OBJETIVOS}

Analisar a distribuição dos principais fatores de risco preexistentes em pacientes diagnosticados com COVID-19 no Estado do Amapá, Amazônia, Brasil.

\section{METODOLOGIA}

Esse estudo propôs-se a fazer uma análise quantitativa e descritiva do número de pacientes acometidos pela COVID-19 no Amapá que atestaram fatores de risco para a doença, realizando-se para isso uma consulta no dia 22 de maio de 2020 no Painel Coronavirus Amapá (painel.corona.ap.gov.br).

Na página principal do Painel Coronavirus Amapá, foi clicado em "baixar CSV" para obtenção dos dados principais dos casos de COVID-19 no Estado do Amapá. Em seguida, no quadro "Comorbidades de pacientes confirmados" da mesma página, selecionou-se a opção "Inspect" no canto superior direito para gerar uma lista das comorbidades e sua relação numérica. O mesmo processo foi repetido no quadro "Comorbidades de Pacientes em Óbito". Posteriormente, para cada uma das listas obtidas, selecionou-se a opção "Fromatted CSV" no campo "Download CSV" para obter os arquivos e utilizá-los no Microsoft Excel.

Dentro desses arquivos, algumas comorbidades e situações de risco foram unidas em um mesmo conjunto com o intuito de uniformizar os resultados. A saber: estão agrupadas -em Doenças Cardíacas Crônicas- doenças cardíacas crônicas, doença cardíaca crônica, doenças cardíacas crônicas, doenças cardíacas crônicas, doença cardiovascular, doença cardíaca, cardiopata, doença cardíaca crônicas, cardíaca; também estão em - Doenças Respiratórias Crônicas - doenças respiratórias crônicas, doença respiratória crônica, doenças respiratórias crônicas, doença respiratória crônica descompensada; Ainda estão em - Hipertensão- hipertensão, HAS, 
hipertenso; novamente estão em - Doença Cardíaca e Hipertensão- Doenças cardíacas crônicas (HAS), doenças cardíacas crônicas - HAS, doença cardiovascular incluindo hipertensão, doença cardíacas crônicas HAS, doença cardíaca (HAS), doença cardíacas crônicas - HAS; bem como estão em - Imunossupressãoimunossupressão, portador de doença cromossômicas ou estado de FR; Novamente estão em - Doença Cardíaca e Diabetes - doenças cardíacas crônicas e diabetes, cardíaca e diabética; Assim estão em - Gestação de Alto Risco - gestante de alto risco, gestante de risco; Por fim estão em - Doenças Renais Crônicas em Estágio Avançado - doença renais crônica em estágio avançados, doença renais crônicas em estágio avançado, doença renais crônica em estágio avançado.

Na planilha "Comorbidades de Pacientes em Óbito", o número de casos registrados como "Sem informação" foram desconsiderados devido a inconclusividade desse recorte. Os demais dados foram selecionados e agrupados conforme descrito anteriormente.

O presente estudo utilizou dados secundários disponíveis em domínio de acesso público e levou em consideração em aspectos éticos norteados pela Resolução $466 / 12$.

\section{RESULTADOS}

Dentre os 28.927 casos confirmados de COVID-19 no Amapá que foram notificados até o dia da coleta, aproximadamente $1,31 \%$ dos pacientes (378 casos) possuíam um fator de risco que poderiam suscetibilizá-los a desenvolver complicações em virtude do acometimento por SARS-CoV-2, conforme visualizado na figura 1.

Figura 1 - Presença de fatores de risco em pacientes diagnosticados com a COVID19 no Amapá até 22 de maio de 2020. 


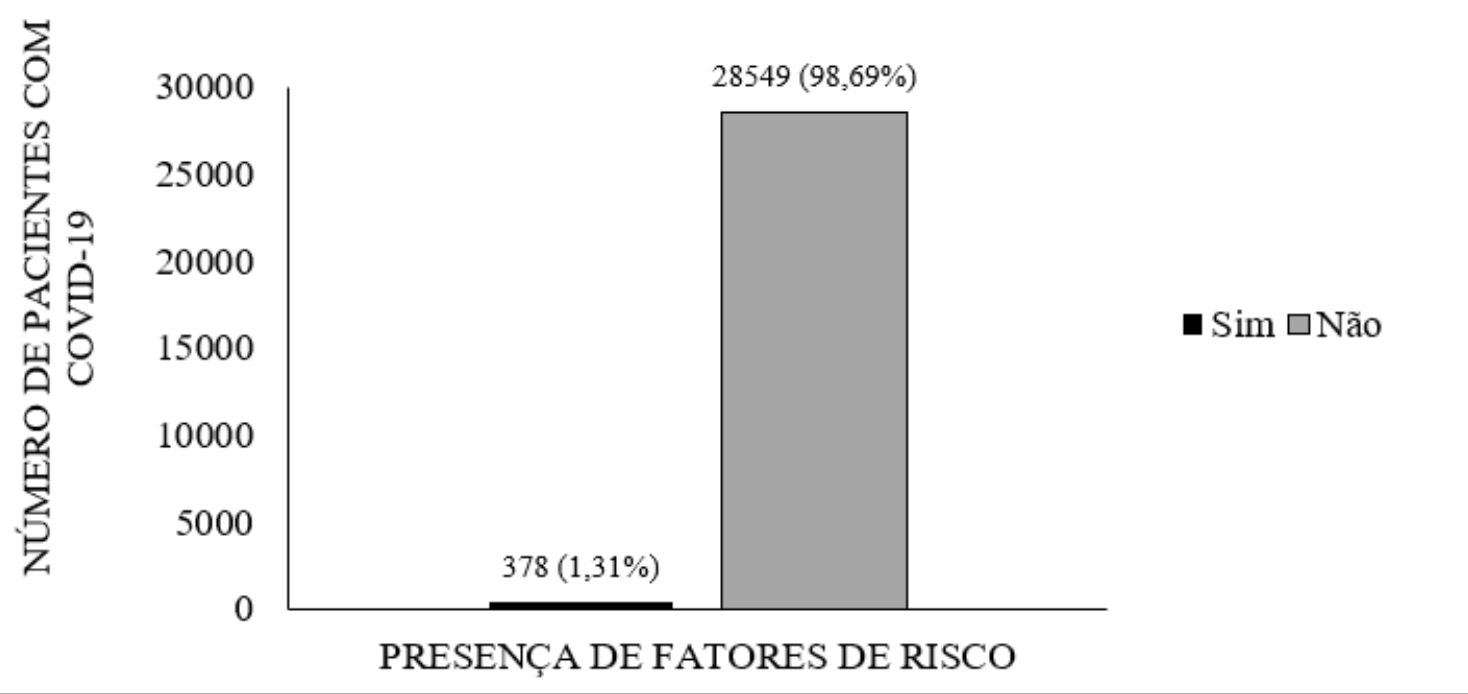

Fonte: Painel Coronavírus Amapá.

Quanto aos pacientes que possuíam fatores de risco, 5,02\% deles (19 casos) possuíam mais de um. Dentre as associações encontradas, incluem-se doença cardíaca e hipertensão $(3,44 \%)$, doença cardíaca e diabetes $(1,06 \%)$, angioplastia e ateromatose coronária $(0,26 \%)$ e doença renal crônica e diabetes $(0,26 \%)$.

Referente aos principais fatores de risco relatados isoladamente pelos pacientes acometidos, verificou-se que a maioria possuía diabetes $29,89 \%$ (112 casos), seguido por doenças cardíacas crônicas com 28,84\% (109 casos).

Percebe-se que as doenças da esfera cardiológica estão presentes de forma majoritária, tendo em vista que 173 pacientes no total são acometidos por doenças desta classe, somando-se os indivíduos com múltiplos fatores de risco e indivíduos somente cardiopatas. 
Tabela 1 - Distribuição dos fatores de risco em pacientes diagnosticados com COVID19 no Amapá.

\begin{tabular}{|l|c|c|}
\hline Fatores de risco & $\begin{array}{c}\text { Número de } \\
\text { relatos }\end{array}$ & Porcentagem(\%) \\
\hline Diabetes & 113 & $29,89 \%$ \\
\hline Doenças Cardíacas Crônicas & 109 & $28,84 \%$ \\
\hline Doenças Respiratória Crônicas & 71 & $18,78 \%$ \\
\hline Hipertensão & 46 & $12,17 \%$ \\
\hline Doenças Cardíacas e Hipertensão & 13 & $3,44 \%$ \\
\hline Imunossupressão & 11 & $2,91 \%$ \\
\hline Doenças Cardíacas e Diabetes & 4 & $1,06 \%$ \\
\hline Gestação de Alto Risco & 4 & $1,06 \%$ \\
\hline Doenças Renais Crônicas em Estágio & 3 & $0,79 \%$ \\
\hline Avançado & & \\
\hline Angioplastia e Ateromatose Coronariana & 1 & $0,26 \%$ \\
\hline Doenças Renais Crônicas e Diabetes & 1 & $0,26 \%$ \\
\hline Cirrose Hepática & 1 & $0,26 \%$ \\
\hline
\end{tabular}




\begin{tabular}{|l|c|c|}
\hline Depressão & 1 & $0,26 \%$ \\
\hline Total & $\mathbf{3 7 8}$ & $\mathbf{1 0 0 , 0 0 \%}$ \\
\hline
\end{tabular}

Fonte: Painel Coronavírus Amapá.

No total, 420 óbitos foram registrados no momento da pesquisa. Deste quantitativo, constatou-se que 318 pacientes possuíam um histórico de comorbidade ou condição predisponente a complicações.

Figura 2 - Presença de fatores de risco nos pacientes que vieram a óbito com a COVID-19 no Amapá até 22 de maio de 2020.

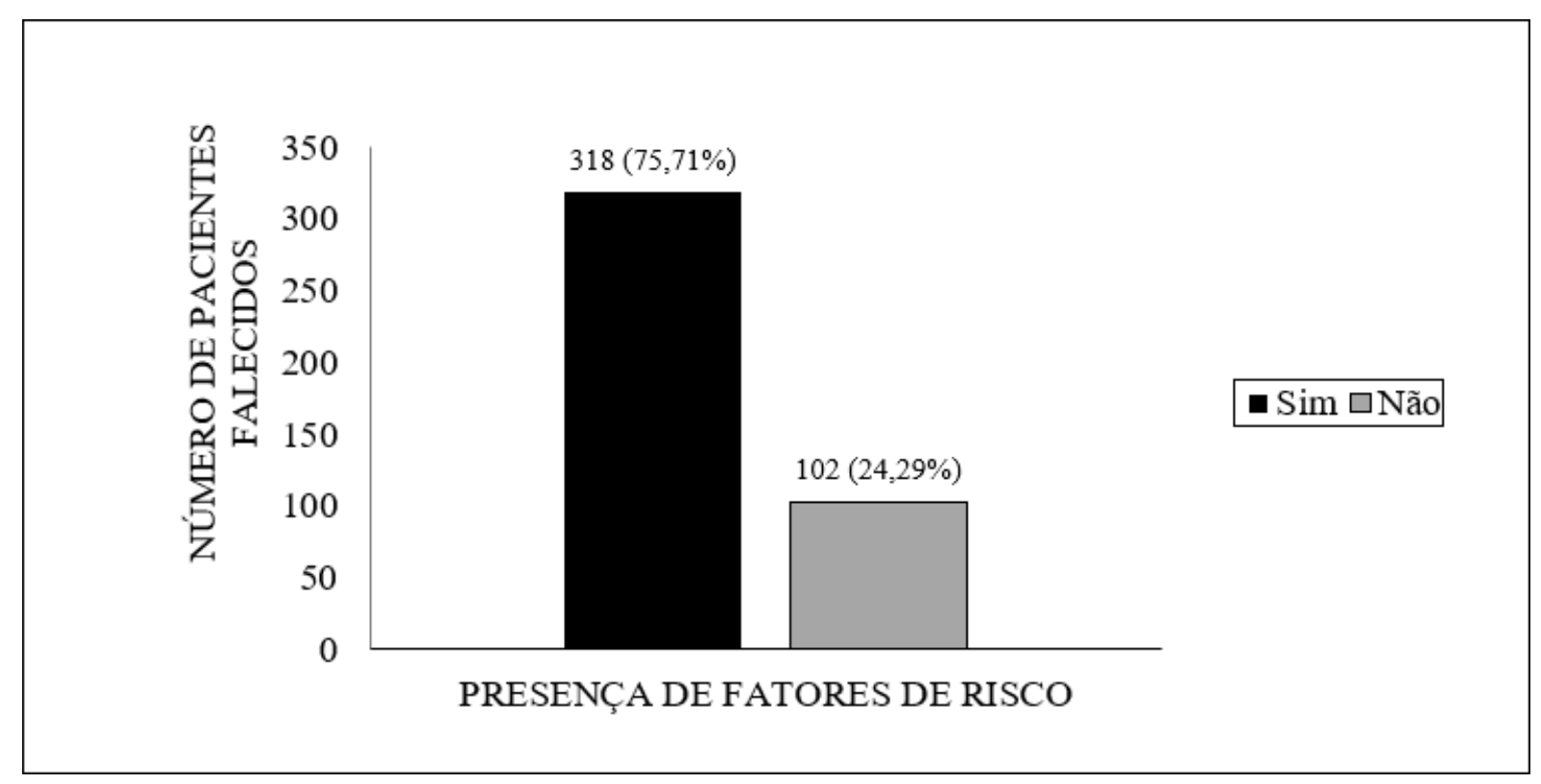

Fonte: Painel Coronavírus Amapá.

Com relação aos fatores de risco presentes em pacientes que vieram a óbito, o principal registro era a hipertensão, presente em $38,68 \%$ dos pacientes (123 casos). Notificou-se também a presença de outros agravantes que não estavam descritos 
anteriormente nos indivíduos com infecção confirmada. São eles obesidade, doença cerebrovascular, neoplasia, tabagismo, Alzheimer, doença do pâncreas, dengue, etilismo e período puerperal.

Tabela 2 - Distribuição de fatores de risco registrados em pacientes que vieram a óbito com COVID-19 no Amapá.

\begin{tabular}{|l|c|c|}
\hline Comorbidade & Número de óbitos & Porcentagem (\%) \\
\hline Hipertensão & 123 & $38,68 \%$ \\
\hline Diabetes & 93 & $29,25 \%$ \\
\hline Doenças Renais & 27 & $8,49 \%$ \\
\hline Cardiopatias & 19 & $5,97 \%$ \\
\hline Obesidade & 19 & $5,97 \%$ \\
\hline Doenças Respiratórias & 15 & $4,72 \%$ \\
\hline Doenças Cerebrovasculares & 8 & $2,52 \%$ \\
\hline Doenças Imunossupressoras & 3 & $0,94 \%$ \\
\hline Neoplasias & 3 & $0,94 \%$ \\
\hline Tabagismo & 2 & $0,63 \%$ \\
\hline Alzheimer & 1 & $0,31 \%$ \\
\hline Doença do Pâncreas & 1 & $0,31 \%$ \\
\hline Doença Hepática & 1 & $0,31 \%$ \\
\hline Dengue & 1 & $0,31 \%$ \\
\hline Etilista & 1 & $0,31 \%$ \\
\hline
\end{tabular}




\section{Puérpera}

Total

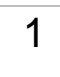

318
$0,31 \%$

$100,00 \%$

Fonte: Painel Coronavírus Amapá.

\section{DISCUSSÃO}

Os estados da região amazônica do Brasil possuem discrepâncias socioeconômicas consideráveis quando comparados aos demais, situação que pode refletir no maior impacto ao sistema de saúde observado. No Estado do Amapá, após a notificação do primeiro caso em 25 de março de 2020, a COVID-19 disseminou-se massivamente, assim como nas demais unidades federativas (DIAS et al., 2020; MENDONÇA et al., 2020).

O número de pacientes com COVID-19 que possuem fatores de risco para a doença no Amapá, comparado ao número total de infectados, é considerado baixo. É consenso na literatura que comorbidades e situações de vulnerabilidade imunológica são considerados fatores agravantes para o quadro de COVID-19, e esse fato pode ter instigado aos indivíduos dos grupos de risco a redobrarem seus cuidados (COSTA et al., 2020b; RODRíGUEZ-COLA et al., 2020).

Em um estudo conduzido por Wang et al. (2020), relatou-se que, de 36 pacientes que necessitaram de atendimento na Unidade de Terapia Intensiva (UTI), 26 (72,2\%) possuíam comorbidades, constatação que indica que um histórico de doenças crônicas pode acarretar no desenvolvimento de complicações. Silva et al. (2020), em um estudo de caracterização clínica e epidemiológica com 1560 pacientes diagnosticados com COVID-19 da cidade de Macapá-AP, constatou que 102 pacientes $(6,54 \%)$ possuíam comorbidades.

As principais comorbidades registradas isoladamente nos pacientes do presente estudo foram diabetes, doenças cardíacas crônicas, doenças respiratórias crônicas e hipertensão. Uma meta-análise feita com um total de 1576 participantes por Yang et al. (2020) destaca também hipertensão $(21,1 \%)$, diabetes $(9,7 \%)$, doenças 
cardiovasculares (8,4\%) e doenças do sistema respiratório $(1,5 \%)$ como as comorbidades mais frequentes. Silva et al. (2020) constataram que, dos 102 pacientes com comorbidades diagnosticados com COVID-19 avaliados, doenças cardiovasculares crônicas (38,33\%), diabetes (24,16\%) e doenças respiratórias crônicas (13,33\%) foram os fatores risco mais prevalentes. Essas condições são associadas a um alto risco de manifestações graves da COVID-19 (GOMES; GOMEZ, 2020; MARTINS-CHAVES), o que se explica em partes pela expressão aumentada da enzima conversora da angiotensina 2 (ECA2) nas células epiteliais alveolares, cardíacas, $\beta$-pancreáticas e do endotélio vascular. A ECA2 permite a entrada do vírus nas células do corpo, fato que corrobora para a falência dos órgãos correspondentes durante o quadro infeccioso (ANGHEBEM; REGO; PICHETH, 2020; ASKIN; TANRIVERDI; ASKIN, 2020).

Alguns pacientes também apresentaram outras comorbidades e fatores de risco, incluindo imunossupressão, gestação de alto risco, doenças renais crônicas, realização de angioplastia com presença de ateromatose coronariana, cirrose hepática e depressão.

Os dados do presente estudo mostram uma letalidade para pacientes com doenças imunossupressoras de $27,27 \%$. Acredita-se que quanto mais comprometido estiver a imunidade do paciente com COVID-19, pior será o prognóstico da doença, motivo que enquadra pacientes imunossuprimidos como grupos de risco para a doença (GOMES; GOMEZ, 2020; MARTINS-CHAVES). No entanto, há relato na literatura de que boa parte dos pacientes imunossuprimidos avaliados seguiram com um bom prognóstico. Um estudo conduzido por Hrusak et al. (2020) com pacientes pediátricos em tratamento anticâncer mostrou que, de 9 crianças diagnosticadas com a COVID-19, 8 seguiram com sintomas leves ou mesmo estavam assintomáticas. Além disso, Bussalino et al. (2020) afirmam que a manutenção da imunossupressão pode tornar brando o curso da COVID-19 para o paciente ao reduzir a tempestade de citocinas característica da doença.

Acerca da gestação, 4 casos de gestação de alto risco foram notificados no Estado. Suspeita-se que hajam riscos maternos pois alterações imunológicas sistêmicas e 
locais são desencadeadas no organismo da mãe, variando de um estado próinflamatório a um anti-inflamatório (LIU et al., 2020). Tratando-se de gestantes com COVID-19, uma meta-análise feita por Trippela et al. (2020) com 275 mulheres grávidas acometidas pela doença mostrou que 269 descreveram sua sintomatologia, sendo que $91,82 \%$ apresentaram sintomas, incluindo febre e tosse como os mais comuns, e 8,18\% encontraram-se assintomáticas. A maior parte das pacientes apresenta sintomas leves ou são assintomáticas, com poucos relatos sobre o desenvolvimento de complicações (CAPARROS-GONZALEZ, 2020; TRIPPELA et al., 2020).

Em relação às doenças renais crônicas, foram notificados somente 3 casos confirmados ao sistema de vigilância epidemiológica do coronavírus do Amapá. Todavia, 27 casos de óbitos de pacientes que possuíam previamente comorbidades dessa classe de doenças foram inseridos no sistema, o que sugere uma possível subnotificação das comorbidades. Em um estudo de coorte prospectivo produzido por Cheng et al. (2020) com 701 indivíduos acometidos pela COVID-19, percebeu-se que $26,7 \%$ das pessoas analisadas possuíam hematúria e 43,9\% proteinúria. Esses achados clínicos sugerem a presença de problemas renais (MOITINHO et al., 2020). Tendo em vista que SARS-CoV-2 age diretamente no receptor da ECA2 (ASKIN; TANRIVERDI; ASKIN, 2020) e que já se evidenciou uma superexpressão desta enzima nas células tubulares proximais de pacientes com doenças renais crônicas (FAN et al., 2020), acredita-se que o histórico de doenças renais possa agravar o quadro de COVID-19 (RENU; PRASANNA; GOPALAKRISHNAN, 2020). Além disso, tem-se associado a preexistência de doenças renais com o comprometimento da funcionalidade do sistema imunológico do paciente, processo que poderia ser explicado pelo processo inflamatório estabelecido durante os quadros de nefropatia. Isso fomenta a ideia de que essas doenças constituem fatores de risco para a COVID19 (OYELADE; ALQAHTANI; CANCIANI, 2020).

Acerca da relação da angioplastia e ateromatose coronária com a COVID-19 não foram encontrados relatos na literatura que pudessem embasar a associação entre esses fatores de risco e a infecção pelo vírus. 
Dos casos notificados de doenças hepáticas no Estado do Amapá, somente 1 caso de cirrose hepática foi relatado. Foi registrado também o óbito de um paciente que possuía doença hepática prévia, mas não é possível afirmar que se trata do mesmo paciente visto que a doença preexistente não foi especificada. A literatura evidencia que a infecção pelo SARS-CoV-2 envolve também o fígado (AGHAGOLI et al., 2020), sendo a presença de hepatopatias considerada um fator determinante para a gravidade do quadro de COVID-19 (RENU; PRASANNA; GOPALAKRISHNAN, 2020). Uma meta-análise feita por Oyelade, Alqahtani e Canciani (2020) envolvendo 5.595 pacientes apontou uma prevalência de doenças hepáticas em paciente com COVID19 de $3 \%$, sendo que, destes, foi relatado gravidade em $57,33 \%$. O mesmo estudo revelou uma taxa de mortalidade de 17,65\%. Apesar da gravidade observa-se, não se encontrou na literatura uma correlação entre a doença hepática pré-existente e o resultado do quadro de COVID-19.

No único caso de depressão registrado no Estado, destaca-se uma preocupação da comunidade científica acerca da possibilidade de doenças neuropsíquicas constituírem também risco para o quadro de COVID-19. Distúrbios da saúde mental, assim como o estado de sofrimento psicológico, influenciam diretamente no funcionamento do sistema imunológico de um indivíduo, podendo suscetibilizá-lo a infecções virais do trato respiratório, o que evidencia uma possível relação entre o estado emocional do paciente e grau de acometimento pelo SARS-CoV-2 (RAJKUMAR, 2020). No entanto, os efeitos gerados pelo vírus sobre os pacientes em questão, assim como a resposta imunológica gerada pelo organismo, não estão bem elucidados na literatura (TROYER; KOHN; HONG, 2020). Para pacientes com histórico de debilidade da saúde mental, deve-se promover uma maior atenção para o quadro, visto que este pode apresentar recaídas ou piora em meio ao cenário pandêmico instalado e a um possível diagnóstico de COVID-19 (YAO; CHEN; XU, 2020).

Os dados coletados no painel coronavírus Amapá apontam que 76\% dos pacientes que vieram a óbito apresentavam comorbidades ou fatores agravantes preexistentes. Um levantamento epidemiológico feito por Almeida et al. (2020) no Estado do 
Maranhão constatou que dos 100 pacientes diagnosticados com COVID-19 e que vieram a óbito, 78\% apresentavam comorbidades prévias, assumindo um padrão similar ao que foi observado no Amapá.

Observa-se que a Hipertensão é o fator de risco mais registrado entre os pacientes amapaenses que vieram a óbito. No entanto, não é possível determinar a taxa de letalidade para pacientes hipertensos, visto que o número de casos confirmados da doença é inferior ao que foi notificado de falecimento. Apesar disso, a literatura evidencia que essa doença é um dos principais fatores agravantes para o quadro de COVID-19, juntamente com outras doenças cardiovasculares (AGHAGOLI et al., 2020; ALMEIDA et al., 2020; FERRARI, 2020). O mecanismo fisiopatológico proposto para explicar essa relação envolve o sistema renina-angiotensina. Devido a maior ligação do SARS-CoV-2 ao ECA2, a concentração desta enzima é reduzida no hospedeiro, acarretando em níveis elevados de angiotensina II e diminuídos de angiotensina 1-7, efeitos estes que geram vasoconstrição e retenção de sódio e pioram o quadro hipertensivo (TADIC et al., 2020).

No que se refere à relação entre a diabetes e o quadro de COVID-19, percebe-se a possibilidade de o vírus piorar as condições clínicas do paciente, tendo em vista que o SARS-COV-2 promove o aumento da glicemia em pacientes com diabetes tipo 2 (BORNSTEIN et al., 2020). O agravamento no quadro de diabéticos pode estar relacionado com o fato de essa comorbidade diminuir a imunidade natural do organismo (COSTA et al., 2020b; MA; HOLT, 2020). Em relação aos casos notificados dessa doença no Amapá, é perceptível que o número de pacientes confirmados (112) é quantitativamente próximo aos que vieram a óbito (93), corroborando assim com a ideia de que o diabetes pode agravar o quadro de COVID-19 ao ponto de o paciente vir a óbito (COSTA et al., 2020b; RONCON et al., 2020).

Outra característica importante da relação diabetes e SARS-COV-2 são os mecanismos de complicações resultantes da união desses dois fatores, já que indivíduos que possuem diabetes e são infectados por um vírus têm mais riscos de desenvolver complicações (LACOBELLIS, 2020). Uma hipótese sobre a diabetes atuar de forma negativa, em conjunto com o vírus, está relacionada a ECA2, uma vez 
que ela está presente em diversos tecidos e atua como porta de entrada para agentes infecciosos na superfície celular (AGHAGOLI et al., 2020; MA; HOLT, 2020). Ademais, pacientes que fazem utilização de Inibidores da Enzima Conversora de Angiotensina (IECAs) tem maiores chances de desenvolverem complicações devido a maior produção de ECA2, a qual favorece a ação do SARS-CoV-2 (FANG; KARAKIULAKIS; ROTH, 2020; FERRARI, 2020; MA; HOLT, 2020).

As cardiopatias, por sua vez, são condições associadas a piores prognósticos e a altos índices de letalidade (AGHAGOLI et al, 2020). Uma metanálise feita por Huang, Wang e Li (2020) constatou que dos 41 pacientes com COVID-19 avaliados, 6 (15\%) possuíam doenças da esfera cardíaca. De fato, observa-se um número considerável de pacientes com cardiopatias que vieram a óbito no Amapá (19), gerando uma taxa de letalidade de $15,07 \%$. A literatura associa essa gravidade com a presença de receptores da ECA2 no coração, os quais são altamente expressos no órgão, o que pode acarretar no desenvolvimento de lesão miocárdica aguda e dano cardiovascular crônico (AGHAGOLI et al, 2020). Além disso, a idade avançada e o efeito de imunossenescência observados na maioria dos pacientes cardiopatas podem estar associados ao prognóstico do quadro de COVID-19 (DRIGGIN et al., 2020).

Foi observado também um número considerável de paciente falecidos com doenças respiratórias, o que corresponde a uma taxa de letalidade de $21,12 \%$ levando em consideração o número de paciente diagnosticados com COVID-19 que relataram comorbidades preexistentes. A preexistência de doenças respiratórias é associada a quadros graves de COVID-19 (MARTINS-CHAVE; GOMES; GOMEZ, 2020; YANG et al., 2020). Sabe-se que o sistema imunológico responde à infecção pelo SARS-CoV2 com um processo inflamatório marcado pela ação de células imunitárias, necrose tecidual e hiperplasia local, efeitos estes que podem prejudicar o processo de troca gasosa dos pulmões e gerar um quadro de pneumonia grave (GIMENEZ et al., 2020), o que pode justificar o motivo de as doenças respiratórias serem um fator de risco para a COVID-19.

Ainda nas informações disponibilizadas pelo Painel Coronavírus Amapá, observa-se que alguns fatores de risco para a COVID-19 constatados em pacientes que faleceram 
não foram notificados da sessão de fatores de risco de pacientes diagnosticados com a doença, como é o caso da obesidade, doenças cerebrovasculares, neoplasias, tabagismo, mal de Alzheimer, doença do pâncreas, dengue, etilismo e período puerperal. Isso impede que se faça uma análise fidedigna do cenário epidemiológico da localidade. No entanto, alguns aspectos acerca dessas condições foram discutidos na literatura.

Acerca da obesidade, sabe-se há tempos que ela está diretamente associada a prognósticos ruins e recuperação demorada em casos de infecções virais. Essa constatação é atribuída ao estado inflamatório constante característico de indivíduos obesos, sustentado pelos níveis elevados de inflamatórios, o qual contribui para o comprometimento da resposta imunológica gerada por esses pacientes e, consequentemente, para a diminuição da capacidade de combater agentes infecciosos (COSTA et al., 2020b; HUSSAIN et al., 2020; KORAKAS et al., 2020). No contexto de COVID-19, outros fatores associados à obesidade podem ser responsáveis pelas maiores chances de agravamento do quadro clínico, o que inclui mecânica respiratória deficitária, baixa taxa de troca gasosa nos pulmões, aumento da resistência das vias aéreas, redução da força pulmonar e menor volume pulmonar (STEFAN et al., 2020). Uma metanálise conduzida por Hussain et al. (2020) mostrou que dos 2.451 pacientes com Índice de Massa Corporal (IMC) maior que $25 \mathrm{~kg} / \mathrm{m}^{2}$ analisados, 531 faleceram, correspondendo a uma taxa de letalidade de $21,66 \%$. O mesmo estudo também avaliou pacientes com IMC menor que $25 \mathrm{~kg} / \mathrm{m}^{2}$, os quais totalizavam 24.506. Destes, 1701 vieram a óbito, uma taxa de letalidade de 6,94\%. A comparação entre esses valores demonstra que o sobrepeso e a obesidade podem influenciar o prognóstico do paciente.

As doenças cerebrovasculares já foram relatadas em quadros mais graves de COVID19 (WANG et al., 2020). O mecanismo por trás do aumento da gravidade para essa classe de paciente ainda é incerto, apesar de já se sugerir que ele seja similar ao das doenças cardiovasculares (PRANATA et al., 2020).

Quanto a relação entre neoplasias e COVID-19, acredita-se que pacientes oncológicos estejam suscetíveis ao desenvolvimento de complicações devido ao 
enfraquecimento da sua atividade imunológica (ASOKAN; RABADIA; YANG, 2020; AL-QUTEIMAT; AMER, 2020). De fato, um estudo retrospectivo feito por Zhang et al. (2020) mostrou que, de 28 pacientes oncológicos avaliados, 8 faleceram (taxa de mortalidade de 28,6\%), indicando um prognóstico ruim para paciente com preexistência de neoplasias.

O histórico de tabagismo por sua vez é especulado como um importante fator de risco para o desenvolvimento de quadros graves da doença, visto que o hábito de fumar está associado ao comprometimento da saúde pulmonar (VARDAVAS; NIKITARA, 2020). Apesar de apenas um caso de tabagismo ser relatados em pacientes que vieram a óbito em nossa análise epidemiológica, existem estudos que demonstram um risco aumentado de gravidade em pacientes com histórico de tabagismo. Alqahtani et al. (2020), em uma metanálise envolvendo 139 fumantes atuais e 28 ex-fumantes diagnosticados com COVID-19, constatou que 31 fumantes atuais (22,30\%) e 13 exfumantes (46\%) apresentaram agravamento de seus quadros clínicos. No mesmo estudo, 5 fumantes atuais dos 13 que desenvolveram complicações vieram a óbito (taxa de mortalidade de 38,5\%). Essas observações apenas reforçam que indivíduos que já fumaram ou fumam devem evitar a prática em meio a pandemia e sempre adotar medidas preventivas.

Sobre o mal de Alzheimer, a associação entre idade avançada e demência, características marcantes da doença, assim como o possível comprometimento do desenvolvimento neurocognitivo desses indivíduos durante o isolamento social na pandemia instigam a comunidade científica a avaliar a preexistência de Alzheimer como fator de risco para a COVID-19 (BRASIL, 2020b; FERINI-STRAMBI; SALSONE, 2020). Tratando-se de mortalidade, Bianchetti et al. (2020) constataram que, de 82 pacientes diagnosticado com a COVID-19 e que apresentavam demência, 51 (62,2\%) morreram, taxa essa superior a de $26,2 \%$ observada em pacientes que não possuía demência, o que salienta que não só o mal de Alzheimer como também outras demências podem estar associadas a um quadro grave de COVID-19.

O caso notificado de um paciente etilista que veio a óbito sugere o hábito como um possível fator de risco para a doença. Essa associação é sustentada pelo 
conhecimento prévio de que o abuso do consumo de álcool pode comprometer a função hepática (EHRMANN; URBAN; DVORAN, 2019), o que suscetibilizaria o paciente a desenvolver complicações e, consequentemente, um quadro mais severo de COVID-19.

A relação entre puerpério e COVID-19 não é tão bem estabelecida quando comparada à gravidez (AN et al., 2020). Entretanto, Tutiya et al. (2020) relataram dois casos de formação de microtrombos pulmonares em puérperas diagnosticadas com COVID-19. Além disso, An et al. (2020) relataram dois casos de puérperas que apresentaram desconforto respiratório e agravamento do quadro após a realização do parto. Essas observações reforçam a necessidade de acompanhar o quadro das pacientes após 0 parto até que haja estabilização.

A dengue e as doenças pancreáticas que foram relatadas nos óbitos por COVID-19 no Amapá, no entanto, não foram encontrados relatos na literatura sobre a associação dessas doenças e o desenvolvimento de complicações do quadro da infecção viral, apesar de que doenças que comprometem a imunidade do paciente tendem aumentar as chances de agravamento de quadros pré-existentes (MARTINS-CHAVES; GOMES; GOMEZ, 2020).

\section{CONCLUSÃO}

A preexistência de comorbidades ou outras condições que prejudiquem a resposta imunológica do organismo constituem potencial fator agravante do quadro infeccioso gerado pelo novo coronavírus, podendo levar o paciente ao óbito. No Estado do Amapá, uma grande parte da população de pacientes diagnosticados com a doença e que faleceram apresentavam um histórico de fator de risco para a COVID-19.

As principais comorbidades relatadas em pacientes diagnosticados com a doença foram diabetes, doenças cardíacas crônicas, doenças respiratórias crônicas e hipertensão. A comorbidade com maior registro nos casos de óbito foi a hipertensão.

A notificação de fatores de risco no quadro de óbito que não foram registrados no quadro de pacientes diagnosticados, assim como a inespecificidade inerente ao 
quadro dos pacientes monitorados pelos mecanismos sanitários do Estado inviabilizaram uma análise mais detalhada acerca do grau de complicação e taxa de letalidade gerados por determinada comorbidade quando da sua associação à infecção por coronavírus.

Para maior completude e fidedignidade dos resultados de um estudo como este, é imprescindível que se tenham dados suficientemente precisos e coerentes, de tal modo que a passagem dos pacientes pela rede de saúde possa ser analisada e então incluída nas pesquisas científicas, tornando possível compreender e enfrentar a atual pandemia e suas repercussões no Amapá.

\section{REFERÊNCIAS}

AGHAGOLI, G. et al. Cardiac involvement in COVID-19 patients: Risk factors, predictors, and complications: A review. J Card Sug., v. 35, p. 1302-1305, abr. 2020.

ALMEIDA, J. S. et al. Caracterização epidemiológica dos casos de covid-19 no maranhão: uma breve análise. Revista Prevenção de Infecção e Sáude, v. 6, mai. 2020.

AL-QUTEIMAT, O. M.; AMER, A. M. The Impact of the COVID-19 Pandemic on Cancer Patients. Am J Clin Oncol., abr. 2020. DOI: 10.1097/COC.0000000000000712.

ALQAHTANI, J. S. et al. Prevalence, Severity and Mortality associated with COPD and Smoking in patients with COVID-19: A Rapid Systematic Review and Meta-Analysis. PLoS One, v. 15, n. 5, e0233147, mai. 2020.

AN, P. et al. Postpartum exacerbation of antenatal COVID-19 pneumonia in 3 women. CMAJ, v. 192, n. 22, p. 603-606, jun. 2020.

ANGHEBEM, M. I.; REGO, F. G. M.; PICHETH, G. COVID-19 e Diabetes: a relação entre duas pandemias distintas. Revista Brasileira de Análises Clínicas. DOI: 10.21877/2448-3877.20200001. 2020. 
ASKIN, L.; TANRIVERDI, O.; ASKIN, H. S. The Effect of Coronavirus Disease 2019 on Cardiovascular Diseases. Arq. Bras. Cardiol., São Paulo, v. 114, n. 5, p. 817-822, mai. 2020.

ASOKAN, I.; RABADIA, S. V.; YANG, E. H. The COVID-19 Pandemic and its Impact on the Cardio-Oncology Population. Curr Oncol Rep., v. 22, n. 6, mai. 2020.

BIANCHETTI, A. et al. Clinical presentation of COVID-19 in dementia patients. J Nutr Health Aging, mai., 2020. DOI: 10.1007/s12603-020-1389-1.

BORNSTEIN, S. R. et al. Practical recommendations for the management of diabetes in patients with COVID-19. The lancet Diabetes \& endocrinology, v. 9, n. 6, p. 546550, jun. 2020.

BRASIL. Plano de contingência DATASUS: Situação de crise provocada pelo novo coronavírus (covid-19). Versão 1.0, 2020a.

BRASIL. MINISTÉRIO DA SAÚDE. Alzheimer: o que é, causas, sintomas, tratamento, diagnóstico e prevenção. Disponível em: $<$ https://saude.gov.br/saudede-a-z/alzheimer>. Acesso em: 31 jul. 2020b.

BRASIL. MINISTÉRIO DA SAÚDE. Painel de casos de doença pelo coronavírus 2019 (COVID-19) no Brasil pelo Ministério da Saúde. Versão 2.0. Disponível em:< https://covid.saude.gov.br/>. Acesso em: 10 de jul. de 2020c.

BUSSALINO, E. et al. Immunosuppressive therapy maintenance in a kidney transplant recipient SARS-CoV-2 pneumonia: a case report. Am J Transplant., abr. 2020. DOI: 10.1111/ajt.15920.

CAPARRO-GONZALEZ, R. A. COVID-19 in PregnantWomen and Neonates: A Systematic Review of the Literature with Quality Assessment of the Studies. Rev Esp Salud Pública., v. 94, abr. 2020.

CHENG, $Y$ et al. Kidney disease is associated with in-hospital death of patients with COVID-19. Kidney Internacional, v. 97, n. 5, p. 829-838, mar. 2020. 
CHINAZZI, M. et al. The effect of travel restrictions on the spread of the 2019 novel coronavirus (COVID-19) outbreak. Science, v. 368, n. 6489, p. 395-400, mar. 2020.

COSTA, I. B. S. S. et al. O Coração e a COVID-19: O que o Cardiologista Precisa Saber. Arq. Bras. Cardiol., v. 114, n. 5, p. 805-816, mai. 2020a.

COSTA, F. F. et al. Metabolic syndrome and COVID-19: An update on the associated comorbidities and proposed therapies. Diabetes \& Metabolic Syndrome: Clinical Research \& Reviews, v. 14, n. 5, p. 809-814, 2020 b.

DIAS, N. L. et al. Predição da propagação do SARS-CoV-2 no Estado do Amapá, Amazônia, Brasil, por modelagem matemática. Revista Científica Multidisciplinar Núcleo do Conhecimento, vol. 06, ano 05, ed. 05, p 73-95, mai., 2020.

DRIGGIN, E. et al. Cardiovascular Considerations for Patients, Health Care Workers, and Health Systems During the COVID-19 Pandemic. J Am Coll Cardiol., v. 75, n. 18, p. 2352-2371, mai. 2020.

ENGIN, A. B.; ENGIN, E. D.; ENGIN, A. Two important controversial risk factors in SARS-CoV-2 infection: obesity and smoking. Environmental Toxicology and Pharmacology, v. 78, mai. 2020. DOI: 10.1016/j.etap.2020.103411.

ENGSTROM, E. et al. Recomendações para a organização da Atenção Primária à Saúde no SUS no enfrentamento da Covid-19. Observatório Covid-19 Fiocruz, Nota técnica. p. 13, mai. 2020.

EHRMANN, J.; URBAN, O.; DVORAN, P. Alcohol-related liver diseases. Cent Eur J Public Health, v. 27, p. 10-14, dez. 2019.

FAN, C. et al. ACE2 Expression in Kidney and Testis May Cause Kidney and Testis Damage After 2019-nCoV Infection. MedRxiv, fev. 2020. DOI: 10.1101/2020.02.12.20022418. 
FANG, L.; KARAKIULAKIS, G.; ROTH, M. Are patients with hypertension and diabetes mellitus at increased risk for COVID-19 infection?. The Lancet. Respiratory Medicine, v. 8, n. 4, mai. 2020.

FERINI-STRAMBI, L.; SALSONE, M. COVID-19 and neurological disorders: are neurodegenerative or neuroimmunological diseases more vulnerable?. J Neurol., jul. 2020. DOI: $10.1007 / \mathrm{s} 00415-020-10070-8$.

FERRARI, F. COVID-19: Dados Atualizados e sua Relação Com o Sistema Cardiovascular. Arq. Bras. Cardiol., v. 114, n. 5, p. 823-826, mai. 2020. DOI: $10.36660 / a b c .20200215$.

GAUTRET, P. et al. Hydroxychloroquine and azithromycin as a treatment of COVID19: results of an open-label non-randomized clinical trial. International journal of antimicrobial agents, mar. 2020. DOI: 10.1016/j.ijantimicag.2020.105949.

GIMENEZ, V. M. M. et al. Lungs as target of COVID-19 infection: Protective common molecular mechanisms of vitamin $D$ and melatonin as a new potential synergistic treatment. Life Sci., v. 354, ago. 2020. DOI: 10.1016/j.lfs.2020.117808.

GUAN, W. et al. Clinical characteristics of coronavirus disease 2019 in China. New England journal of medicine, v. 382, n. 18, p. 1708-1720, fev. 2020.

HONG, H. et al. Clinical characteristics of novel coronavirus disease 2019 (COVID-19) in newborns, infants and children. Pediatrics and Neonatology, v. 61, n. 2, p. 131 132, abr. 2020.

HRUSAK, O. et al. Flash survey on severe acute respiratory syndrome coronavirus-2 infections in paediatric patients on anticancer treatment. European Journal of Cancer, v. 132, p. 11-16, abr. 2020.

HUANG, C.; WANG, Y.; LI, X. Características clínicas de pacientes infectados com o novo coronavírus de 2019 em Wuhan, China. Lanceta, v. 395, p. 497-506, jan. 2020. 
HUSSAIN, A. Obesity and mortality of COVID-19. Meta-analysis. Obes Res Clin Pract., jul. 2020. DOI: 10.1016/j.orcp.2020.07.002.

KHALIQ, O. P.; PHOSWA, W. N. Is Pregnancy a Risk Factor of COVID-19?. European Journal of Obstetrics \& Gynecology and Reproductive Biology, 2020. DOI: 10.1016/j.ejogrb.2020.06.058.

IACOBELLIS, G. COVID-19 and diabetes: can DPP4 inhibition play a role?. Diabetes research and clinical practice, v. 162, abr. 2020. DOI: 10.1016/j.diabres.2020.108125.

LI, H. et al. Coronavirus disease 2019 (COVID-19): current status and future perspective. International journal of antimicrobial agents, v. 55, n. 5, mai. 2020.

LIU, H. et al. Why are pregnant women susceptible to COVID-19? An immunological Viewpoint. Journal of reproductive immunology, v. 139, jun. 2020.

LIMA, D. L. P. et al. COVID-19 no estado do Ceará, Brasil: comportamentos e crenças na chegada da pandemia. Ciênc. saúde coletiva, v. 25, n. 5, p. 1575-1586, mai. 2020.

LUCENA, T. M. C. et al. Mechanism of inflammatory response in associated comorbidities in COVID-19. Diabetes \& Metabolic Syndrome: Clinical Research \& Reviews, mai. 2020. DOI: 10.1016/j.dsx.2020.05.025.

MA, R. C. W.; HOLT, R. I. G. COVID-19 and diabetes. Diabetic Medicine, abr. 2020. DOI: $10.1111 / \mathrm{dme} .14300$.

MARTINS-CHAVES, R. R.; GOMES, C. C.; GOMEZ, R. S. Immunocompromised patients and coronavirus disease 2019: a review and recommendations for dental health care. Braz. res oral., São Paulo, v. 34, e048, mai. 2020.

MENDONÇA, F. D. Região Norte do Brasil e a pandemia de COVID-19: análise socioeconômica e epidemiológica. Journal Health NPEPS, v. 5, n. 1, 2020. 
MOITINHO, M. S. et al. Lesão renal aguda pelo vírus SARS-COV-2 em pacientes com COVID-19: revisão integrativa. Rev. Bras. Enferm., Brasília, v. 73, n. 2, jul. 2020.

OLIVEIRA, A. C.; LUCAS, T. C.; IQUIAPAZA, R. A. What has the COVID-19 pandemic taught us about adopting preventive measures?. Texto \& Contexto Enfermagem, Florianópolis, v. 29, mai. 2020. DOI: 10.1590/1980-265x-tce-2020-0106.

OLIVEIRA, W. K. et al. Como o Brasil pode deter a COVID-19. Epidemiol. Serv. Saude, Brasília, v. 9, n. 2, p. 1-8, abr. 2020.

OMS. ORGANIZAÇÃO MUDIAL DA SAÚDE. Painel da Doença de Coronavírus da OMS (COVID-19). Disponível em:<https://covid19.who.int/>. Acesso em: 10 de jul. de 2020.

OYELADE, T.; ALQAHTANI, J.; CANCIANI, G. Prognosis of COVID-19 in Patients with Liver and Kidney Diseases: An Early Systematic Review and Meta-Analysis. Trop. Med. Infect. Dis., v. 5, n. 80, mai. 2020.

PRANATA, R. et al. Impact of cerebrovascular and cardiovascular diseases on mortality and severity of COVID-19-systematic review, meta-analysis, and metaregression. J Stroke Cerebrovasc Dis., v. 29, n. 8,: mai. 2020.

PIRES, L. N.; CARVALHO, L.; XAVIER, L. L. COVID-19 e desigualdade: a distribuição dos fatores de risco no Brasil. Research Gate, abr. 2020. DOI: 10.13140/RG.2.2.27014.73282.

RAJKUMAR, R. P. Ayurveda and COVID-19: Where psychoneuroimmunology and the meaning response meet. Brain, behavior and immunity, v. 87, p. 8-9, jul. 2020.

RENU, K.; PRASANNA, P. L.; GOPALAKRISHNAN, A. V. Coronaviruses pathogenesis, comorbidities and multi-organ damage - A review. Life sci., v. 255, mai. 2020. DOI: 10.1016/j.lfs.2020.117839. 
RODRÍGUEZ-COLA, M. et al. Clinical features of coronavirus disease 2019 (COVID19 ) in a cohort of patients with disability due to spinal cord injury. Spinal Cord Series and Cases, v. 6, n. 39, mai. 2020.

RONCON, L. et al. Diabetic patients with COVID-19 infection are at higher risk of ICU admission and poor short-term outcome. Journal of Clinical Virology, v. 127, jun. 2020. DOI: 10.1016 / j.jcv.2020.104354.

RUBIO-PÉREZ, I. et al. COVID-19: Conceptos clave para el cirujano. Cirugía Española, v. 98, n. 6, p. 310-319, jun./jul. 2020.

SIA, S. F. et al. Pathogenesis and transmission of SARS-CoV-2 in golden hamsters. Nature, mai. 2020. DOI: 10.1038/s41586-020-2342-5.

SILVA, A.W.C. et al. Caracterização clínica e epidemiologia de 1560 casos de COVID19 em Macapá/AP, extremo norte do Brasil. Research, Society and Development, v. 9, n.8, e150985499, jun. 2020.

STEFAN. N. et al. Obesity and impaired metabolic health in patients with COVID-19. Nat Rev Endocrinol., abr. 2020. DOI: 10.1038/s41574-020-0364-6.

TAY, M. Z. et al. The trinity of COVID-19: immunity, inflammation and intervention. Nat Rev Immunol, v. 20, p. 363-374, abr. 2020. DOI: 10.1038/s41577-020-0311-8.

TADIC, M. et al. COVID-19 and arterial hypertension: Hypothesis or evidence? J Clin Hypertens (Greenwich), jul. 2020. DOI:10.1111/jch.13925.

TRIPPELA, G. et al. COVID-19 in PregnantWomen and Neonates: A Systematic Review of the Literature with Quality Assessment of the Studies. Pathogens, v. 9, n. 6, jun. 2020.

TROYER, E. A.; KOHN, J. N.; HONG, S. Are we facing a crashing wave of neuropsychiatric sequelae of COVID-19? Neuropsychiatric symptoms and potential immunologic mechanisms. Brain, behavior and immunity, v. 87, p. 34-39, jul. 2020. 
TUTIYA, C. T. et al. Possible formation of pulmonary microthrombi in the early puerperium of pregnant women critically ill with COVID-19: Two case reports. Case Rep Womens Health, v. 27, e00237, jun. 2020.

VARDAVAS, C. I.; NIKITARA, K. COVID-19 and smoking: A systematic review of the evidence. Tob Induc Dis., v. 18, mar. 2020. DOI: 10.18332/tid/119324.

WANG, D. et al. Clinical Characteristics of 138 Hospitalized Patients With 2019 Novel Coronavirus-Infected Pneumonia in Wuhan, China. Journal of American Medical Asssociation, v. 323, n. 11, p. 1061-1069, fev. 2020.

YANG, J. et al. Prevalence of comorbidities and its effects in patients infected with SARS-CoV-2: a systematic review and meta-analysis. Int $\mathbf{J}$ Infect Dis., v. 94, p. 9195, mai. 2020.

YAO, H.; CHEN, J. H.; XU, Y. F. Patients with mental health disorders in the COVID19 epidemic. Lancet Psychiatry, v. 7, n. 4, mar. 2020.

ZHAI, P. et al. The epidemiology, diagnosis and treatment of COVID-19. International journal of antimicrobial agents, v. 55, n. 5, mai. 2020.

ZHANG, L. et al. Clinical characteristics of COVID-19-infected cancer patients: a retrospective case study in three hospitals within Wuhan, China. Ann Oncol., v. 31, n. 7, p 894-901, jul. 2020.

Enviado: Junho, 2021.

Aprovado: Junho, 2021. 